\title{
ON BOUNDARY CRITICAL POINTS FOR SEMIGROUPS OF ANALYTIC FUNCTIONS
}

\author{
M. D. CONTRERAS, S. DÍAZ-MADRIGAL and CH. POMMERENKE*
}

\begin{abstract}
We analyze the relationship between boundary fixed points of semigroups of analytic functions and boundary critical points of their infinitesimal generators. As a consequence, we show two new inequalities for analytic self-maps of the unit disk. The first one is about angular derivatives at fixed points of functions belonging to semigroups of analytic functions. The second one deals with angular derivatives at contact points of arbitrary analytic functions from the unit disk into itself.
\end{abstract}

\section{Introduction and statement of the results}

1. A (one-parameter) semigroup of analytic functions is any continuous homomorphism $\Phi: t \mapsto \Phi(t)=\varphi_{t}$ from the additive semigroup of non-negative real numbers into the composition semigroup of all analytic functions which map $D$ into $D$. That is, $\Phi$ satisfies the following three conditions:

a) $\varphi_{0}$ is the identity in $\mathrm{D}$,

b) $\varphi_{t+s}=\varphi_{t} \circ \varphi_{s}$, for all $t, s \geq 0$,

c) $\varphi_{t}(z)$ tends to $z$ as $t$ tends to 0 , uniformly on compact subsets of D.

These type of semigroups appear in many different areas of Analysis. Among them, we cite the theory of composition operators, the theory of Markov stochastic processes, optimization theory or the theory of planar vector fields. In this paper, we are interested in this last aspect which we expose in detail for the sake of completeness.

Given a semigroup $\Phi=\left(\varphi_{t}\right)$, it is well-known (see [10], [1]) that there exists a unique analytic function $G: \mathrm{D} \rightarrow \mathrm{C}$ such that,

$$
\frac{\partial \varphi_{t}(z)}{\partial t}=G\left(\varphi_{t}(z)\right)=G(z) \frac{\partial \varphi_{t}(z)}{\partial z} \quad \text { for all } z \in \mathrm{D} \text { and } t \geq 0 .
$$

* This research has been partially supported by the Ministerio de Ciencia y Tecnología and the European Union (FEDER) project BFM2003-07294-C02-02 and by La Consejería de Educación y Ciencia de la Junta de Andalucía.

Received January 24, 2005. 
To simplify the notation, we denote $\varphi_{t}^{\prime}(z)=\frac{\partial \varphi_{t}(z)}{\partial z}$. In what follows, $G$ will be called the vector field associated with $\Phi$ or the infinitesimal generator of $\Phi$. There is a very nice representation, due to Berkson and Porta [1], of those analytic functions on the disk which are generated in this way:

An analytic function $G: \mathrm{D} \rightarrow \mathrm{C}$ is the infinitesimal generator of a semigroup of analytic functions $\Phi$ if and only if there is a point $b \in \overline{\mathrm{D}}$ and an analytic function $p: \mathrm{D} \rightarrow \mathrm{C}$ with $\operatorname{Re} p \geq 0$ such that

$$
G(z)=(b-z)(1-\bar{b} z) p(z), \quad z \in \mathrm{D} .
$$

Moreover, such a representation is unique. The point $b$ is called the Denjoy-Wolff point of $\Phi$ and $p$ is called the Carathéodory function associated to $\Phi$.

Looking at the above representation, we see that, whenever $b \in \mathrm{D}$, the associated dynamical system has a critical point. However, $G$ never vanishes if $b \in \partial \mathrm{D}$. So it is natural to wonder about "critical points" belonging to $\partial \mathrm{D}$. Since, in our hypothesis, $G$ and the functions $\left(\varphi_{t}\right)$ are only defined in D, this comment requires a clarification and this leads us to recall some concepts from Complex Function Theory [7], [8].

Let $\varphi: \mathrm{D} \rightarrow \mathrm{C}$ be an analytic function and $a \in \partial \mathrm{D}$. We say that $L \in \mathrm{C}_{\infty}$ is the angular limit of $\varphi$ in $a$ when $z$ tends to $a$ if, for every $\alpha \in(0, \pi / 2)$,

$$
\lim _{z \in S(a, \alpha), z \rightarrow a} \varphi(z)=L,
$$

where $S(a, \alpha)=\{z \in \mathrm{D}:|\operatorname{Arg}(1-\bar{a} z)|<\alpha\}$ denotes the Stolz angle of opening angle $\alpha$ with vertex $a$. The number $L$ is commonly denoted by $\angle \lim _{z \rightarrow a} \varphi(z)$. When $\angle \lim _{z \rightarrow a} \varphi(z)=a$, the point $a$ is called a boundary fixed point of $\varphi$. If we suppose that $\varphi(\mathrm{D}) \subset \mathrm{D}$ and that $\angle \lim _{z \rightarrow a} \varphi(z)=b \in \partial \mathrm{D}$, it is well-known that

$$
\angle \lim _{z \rightarrow a} \frac{\varphi(z)-b}{z-a}
$$

always exists. This value is denoted by $\varphi^{\prime}(a)$. It satisfies that $a \bar{b} \varphi^{\prime}(a) \in$ $(0,+\infty) \cup\{\infty\}$. When $\varphi^{\prime}(a) \neq \infty$, the $\operatorname{limit} \angle \lim _{z \rightarrow a} \varphi^{\prime}(z)$ also exists and is equal to $\varphi^{\prime}(a)$. In particular, if $a$ is a boundary fixed point, we have that $\varphi^{\prime}(a) \in(0,+\infty) \cup\{\infty\}$. Following standard usage in iteration theory, we will say that $a$ is attractive if $\varphi^{\prime}(a) \in(0,1]$; repulsive if $\varphi^{\prime}(a) \in(1,+\infty)$ and superrepulsive if $\varphi^{\prime}(a)=\infty$. These definitions are coherent with the intuitive geometric meaning of a repulsive or an attractive point, thanks to the celebrated Denjoy-Wolff Theorem [9, Section 5.1].

Now, we come back to the semigroup $\Phi=\left(\varphi_{t}\right)$ and its associated vector field $G$. A point $a \in \partial \mathrm{D}$ is called a boundary fixed point of $\Phi$ if $a$ is a boundary 
fixed point for each $\varphi_{t}$, where $t \geq 0$. By [3], "for each $\varphi_{t}$ " can be replaced by "for some $\varphi_{t}(t>0)$ ". What is more, a point $a \in \partial \mathrm{D}$ is attractive (resp. repulsive, superrepulsive) for some $t>0$ if and only if the same happens for all $t$ (see Lemmata 3 and 1 below). It is a non-trivial fact that the Denjoy-Wolff point is common for all the iterates belonging to a non-elliptic semigroup (that is, when the functions of the semigroup have no fixed point in the unit disk).

2. Our main result characterizes boundary critical points of vector fields with finite angular derivatives in terms of non-superrepulsive fixed points.

THEOREM 1. Let $\left(\varphi_{t}\right)$ be a semigroup of analytic functions and denote by $G$ its infinitesimal generator. Let $a \in \partial \mathrm{D}$. Then the following are equivalent:

(1) The point a is a non-superrepulsive fixed point of the semigroup.

(2) The angular limit

$$
\angle \lim _{z \rightarrow a} \frac{G(z)}{z-a}
$$

exists finitely.

(3) The angular limit

$$
\angle \lim _{z \rightarrow a} G(z)=0,
$$

and the angular limit $\angle \lim _{z \rightarrow a} G^{\prime}(z)$ exists finitely.

Moreover if one of the above assertions holds, then

(i) $\angle \lim _{z \rightarrow a} \frac{G(z)}{z-a}=\angle \lim _{z \rightarrow a} G^{\prime}(z) \in \mathbf{R}$.

(ii) If we denote by $\beta:=\angle \lim _{z \rightarrow a} \frac{G(z)}{z-a}$, then

$$
\varphi_{t}^{\prime}(a)=e^{\beta t} \quad \text { for all } t \geq 0 .
$$

It is worth pointing out that it can be proved that whenever $\angle \lim _{z \rightarrow a} G(z)=$ 0 , the limit $\angle \lim _{z \rightarrow a} \frac{G(z)}{z-a}$ always exists in $C_{\infty}$ (it can take the value $\infty$ ). Moreover, it is even possible to have

$$
\angle \lim _{z \rightarrow a} G(z)=0 \quad \text { and } \quad \angle \lim _{z \rightarrow a} \frac{G(z)}{z-a}=\infty,
$$

and, at the same time, $a$ is not a fixed point of the semigroup. For example, consider the semigroup given by

$$
\varphi_{t}(z)=1-\left[1-e^{-t / 2}+e^{-t / 2} \sqrt{1-z}\right]^{2}
$$

for all $t \geq 0$ and $z \in \mathrm{D}$. In this case, the Denjoy-Wolff point is zero and $\left(\varphi_{t}\right)$ has no boundary fixed point. But the infinitesimal generator of this semigroup 
is $G(z)=1-z-\sqrt{1-z}$. Therefore, $\angle \lim _{z \rightarrow 1} G(z)=0$ and $\angle \lim _{z \rightarrow 1} \frac{G(z)}{z-1}=$ $\infty$.

3. In [4], Cowen and Pommerenke showed that if $\varphi$ is an analytic and univalent function in $\mathrm{D}$ with $\varphi(\mathrm{D}) \subset \mathrm{D}$, with Denjoy-Wolff point $b \in \overline{\mathrm{D}}$, and $\xi_{1}, \xi_{2}, \ldots, \xi_{n}$ are distinct fixed points of $\varphi$ (different from $b$ ), then:

a: If $b \in \partial \mathrm{D}$, then

$$
\sum_{j=1}^{n} \frac{1}{\log \varphi^{\prime}\left(\xi_{j}\right)} \leq-\frac{1}{\log \varphi^{\prime}(b)}
$$

Moreover, equality holds if and only if

$$
\varphi(z)=\sigma^{-1}(\sigma(z)+1)
$$

where

$$
\sigma(z)=\sum_{j=1}^{n} \frac{1}{\log \varphi^{\prime}\left(\xi_{j}\right)} \log \left[\frac{b+z}{b-z}-\frac{b+\xi_{j}}{b-\xi_{j}}\right]
$$

b: If $b=0$, then

$$
\sum_{j=1}^{n} \frac{1}{\log \varphi^{\prime}\left(\xi_{j}\right)} \leq 2 \operatorname{Re} \frac{1}{B}
$$

where $B=\lim _{r \rightarrow 1^{-}} \log \frac{\varphi\left(r \xi_{j}\right)}{\varphi^{\prime}(0) r \xi_{j}}$ (this limit is independent of $j$, we choose the branch of $\log \frac{\varphi(z)}{\varphi^{\prime}(0) z}$ that is zero at $\left.z=0\right)$. Moreover, equality holds if and only if

$$
\varphi(z)=\sigma^{-1}\left(\varphi^{\prime}(0) \sigma(z)\right)
$$

where

$$
\sigma(z)=z \prod_{j=1}^{n}\left(1-\overline{\xi_{j}} z\right)^{-\frac{B}{\log \varphi^{\prime}\left(\xi_{j}\right)}}
$$

Note that the inequality (a) says nothing if $\varphi^{\prime}(b)=1$. In [4, Theorem 6.1], they also obtained another inequality for the case $\varphi^{\prime}(b)=1$. However the authors remarked that is not the best possible and in some particular cases gives no information (see [4, page 284]). This problem was also considered by $\mathrm{Li}$ [5] who used a different approach. Using the above theorem we can give a new sharp inequality for those functions which can be embedded in a semigroup of analytic functions.

THEOREM 2. Let $\left(\varphi_{t}\right)$ be a semigroup of analytic functions with DenjoyWolff point $b \in \partial \mathrm{D}$ and denote by $G$ its infinitesimal generator. If $\xi_{1}, \xi_{2}, \ldots, \xi_{n}$ 
are distinct repulsive fixed points of $\varphi$ (different from $b$ ) with $\varphi^{\prime}\left(\xi_{j}\right)=e^{\beta_{j} t}$ for all $j$ and for all $t \geq 0$ (note that $\beta_{j} \in(0,+\infty)$ ) then

$$
\sum_{j=1}^{n} \frac{1-\operatorname{Re}\left(\bar{b} \xi_{j}\right)}{\beta_{j}} \leq \operatorname{Re} \frac{1}{\bar{b} G(0)} .
$$

Moreover, equality holds if and only if

$$
\varphi_{t}(z)=\sigma^{-1}(\sigma(z)+t)
$$

where

$$
\begin{aligned}
\sigma(z)= & \frac{z}{(1-\bar{b} z) G(0)} \\
& +2 \sum_{j=1}^{n} \frac{1-\operatorname{Re}\left(\bar{b} \xi_{j}\right)}{\beta_{j}}\left[\frac{\bar{b} \xi_{j}}{\left(1-\bar{b} \xi_{j}\right)^{2}} \log \left[\frac{1-\bar{b} z}{1-\overline{\xi_{j}} z}\right]+\frac{\overline{\xi_{j}}}{1-\overline{\xi_{j}} b} \frac{z}{1-\bar{b} z}\right] .
\end{aligned}
$$

4. We finish the paper giving some applications of Theorem 1 to inequalities involving points of the set $\varphi^{-1}(\lambda)$. In [4], Cowen and Pommerenke proved that if $\varphi$ is analytic in $\mathbf{D}$ with $\varphi(\mathrm{D}) \subset \mathrm{D}, \lambda \in \partial \mathrm{D}$ and $z_{1}, z_{2}, \ldots, z_{n}$ are distinct points of $\partial \mathrm{D}$ with $\varphi\left(z_{j}\right)=\lambda$ for all $j=1, \ldots, n$, then

$$
\sum_{j=1}^{n} \frac{1}{\left|\varphi^{\prime}\left(z_{j}\right)\right|} \leq \operatorname{Re} \frac{\lambda+\varphi(0)}{\lambda-\varphi(0)}
$$

and equality holds if and only if $\varphi$ is a Blaschke product of order $n$. Now we present another inequality.

Theorem 3. Let $\varphi$ be an analytic in $\mathrm{D}$ with $\varphi(\mathrm{D}) \subset \mathrm{D}, b, a \in \partial \mathrm{D}$, with $\varphi(b)=a, \lambda \in \partial \mathbf{D}$, with $\lambda \neq a$, and $z_{1}, z_{2}, \ldots, z_{n}$ are distinct points of $\partial \mathrm{D}$ with $\varphi\left(z_{j}\right)=\lambda$ for all $j=1, \ldots, n$, then

$$
\sum_{j=1}^{n} \frac{1-\operatorname{Re}(\bar{\lambda} a)}{1-\operatorname{Re}\left(\overline{z_{j}} b\right)} \frac{1}{\left|\varphi^{\prime}\left(z_{j}\right)\right|} \leq\left|\varphi^{\prime}(b)\right|
$$

and equality holds if and only if

$$
\varphi(z)=\frac{1+(1-\lambda \bar{a}) \sum_{j=1}^{n} \frac{1}{\left|\varphi^{\prime}\left(z_{j}\right)\right|} \frac{z_{j}}{b-z_{j}} \frac{z-b}{z-z_{j}}}{1-(1-\lambda \bar{a}) \sum_{j=1}^{n} \frac{1}{\left|\varphi^{\prime}\left(z_{j}\right)\right|} \frac{z_{j}}{b-z_{j}} \frac{z-b}{z-z_{j}}}
$$

for all $z \in \mathrm{D}$. 


\section{Proofs}

The proof of our main theorem depends on considering a model flow where the trajectories become straight lines or spirals but they fill in a more involved planar domain $\Omega$. This domain $\Omega$ is constructed by means of the theory of univalent functions [1]. If the Denjoy-Wolff point $b$ of the semigroup belongs to $\partial \mathrm{D}$, then there is a unique univalent function $\sigma: \mathrm{D} \rightarrow \mathrm{C}$ with $\sigma(0)=0$ verifying the property

$$
\Omega+t \subset \Omega, \text { for each } t>0 \text {, where } \Omega:=\sigma(\mathrm{D}),
$$

and such that

$$
\varphi_{t}(z)=b \sigma^{-1}(\sigma(\bar{b} z)+t), \quad t \geq 0, \quad z \in \mathrm{D} .
$$

Then $\Omega:=\sigma(\mathrm{D}) \subset \mathrm{C}$ is called the associated planar domain of $\Phi$. In this case, we have that $G(z)=\frac{b}{\sigma^{\prime}(\bar{b} z)}$ for all $z \in \mathrm{D}$.

Likewise, if the Denjoy-Wolff point $b$ belongs to $\mathrm{D}$, then there is a unique univalent function $\sigma: \mathrm{D} \rightarrow \mathrm{C}$ with $\sigma(0)=0$ and $\sigma^{\prime}(0)=1$, verifying the geometric condition

"there is $c \in \mathrm{C}$ with $\operatorname{Re}(c)>0$ such that, for each $t>0, e^{-c t} \Omega \subset \Omega$

where $\Omega:=\sigma(\mathrm{D})$ "

and such that

$$
\varphi_{t}(z)=\sigma_{b}^{-1}\left(e^{-c t} \sigma_{b}(z)\right), \quad t \geq 0, \quad z \in \mathrm{D},
$$

where $\sigma_{b}=\sigma \circ m_{b}$. As usual, $m_{b}$ is the elliptic automorphism of the disk associated to $b$, that is,

$$
m_{b}(z)=\frac{b-z}{1-\bar{b} z}, \quad z \in \mathrm{D} .
$$

The set $\Omega=\sigma(\mathrm{D}) \subset \mathrm{C}$ is again called the associated planar domain of $\Phi$ and, in this case, we have that $G(z)=-c \frac{\sigma_{b}(z)}{\sigma_{b}^{\prime}(z)}$ for all $z \in \mathrm{D}$.

Lemma 1. Let $\left(\varphi_{t}\right)$ be a semigroup of analytic functions with Denjoy-Wolff point equal to 1 . Suppose that a is a fixed point of $\left(\varphi_{t}\right)$. Then there exists $\beta \in \mathbf{R} \cup\{\infty\}$ such that $\varphi_{t}^{\prime}(a)=e^{\beta t}$ for all $t>0$. If $\beta \in(0,+\infty)$, then there is a real number $c$ such that the open strip

$$
V=\left\{z \in \mathrm{C}: c-\frac{\pi}{2 \beta}<\operatorname{Im} z<c+\frac{\pi}{2 \beta}\right\}
$$


is contained in $\Omega$ and the curve $\gamma(t)=\sigma^{-1}(w-t)$ converges to a nontangentially as $t$ goes to $+\infty$ for all $w \in V$. Moreover, given a curve $\gamma$ : $[0,1) \rightarrow D$ such that $\gamma(r)$ converges to a nontangentially, then there exist $r_{0} \in(0,1)$ and $\varepsilon>0$ such that for all $r \geq r_{0}$, we have that $\sigma(\gamma(r)) \in V$ and $\operatorname{dist}(\sigma(\gamma(r)), \partial V)>\varepsilon$.

The proof of this lemma can be found in [2, Lemma 20] when $\gamma(r)=r a$, but the proof can be easily adapted to this nontangential case.

Lemma 2. Let $g, h: \mathrm{D} \rightarrow \mathrm{D}$ be two analytic functions and consider $f=$ $g \circ h$. If $f(1)=h(1)=1$, then $g(1)=1$ and $f^{\prime}(1)=g^{\prime}(1) h^{\prime}(1)$ (note that derivatives could be infinite).

Proof. The curve $C=\{h(r): 0 \leq r<1\}$ ends in $h(1)=1$. We have that

$$
g(h(r))=f(r) \rightarrow 1 \quad \text { as } \quad r \rightarrow 1 .
$$

That is, $\lim _{z \rightarrow 1, z \in C} g(z)=1$. Therefore, by the Lehto-Virtanen Theorem [8, Chapter 4], we know that $g(1)=1$. Moreover, we have that

$$
\frac{1-f(r)}{1-r}=\frac{1-g(h(r))}{1-h(r)} \frac{1-h(r)}{1-r} .
$$

If $h^{\prime}(1)$ is finite, then $C$ is in a Stolz angle and, by (2.1), we have that $f^{\prime}(1)=$ $g^{\prime}(1) h^{\prime}(1)$.

Now, suppose that $h^{\prime}(1)=\infty$ and $f^{\prime}(1)$ is finite. Consider the function

$$
p(z)=\frac{1-g(z)}{1-z} \quad \text { for all } \quad z \in \mathrm{D} .
$$

Since $f^{\prime}(1)$ is finite and $h^{\prime}(1)=\infty$, we have that $\lim _{z \rightarrow 1, z \in C} p(z)=0$. Moreover, we have that $p(z) \notin(-\infty, 0]$ for all $z \in \mathrm{D}$. So, we have that $|\operatorname{Im} \log p(z)|<\pi$ for all $z \in \mathrm{D}$. Therefore, $\log p$ is a Bloch function and $p$ is normal. Now, we can apply the Lehto-Virtanen Theorem to obtain that $g^{\prime}(1)=p(1)=0$. But, by the Julia-Wolff Lemma, $g^{\prime}(1)>0$. Therefore, if $h^{\prime}(1)=\infty$ then $f^{\prime}(1)=\infty$ and we are done.

Lemma 3. Let $\left(\varphi_{t}\right)$ be a semigroup of analytic functions with Denjoy-Wolff point equal to 0 . Suppose that a is a fixed point of $\left(\varphi_{t}\right)$. Then there exists $\beta \in(0,+\infty) \cup\{\infty\}$ such that $\varphi_{t}^{\prime}(a)=e^{\beta t}$ for all $t>0$. If $\beta \in(0,+\infty)$, then there are a real number $\theta_{0}$ and $d \in \partial \mathrm{D}$ such that the open spiral sector

$$
V=\left\{w \in \mathbf{C}: w=d e^{-c t} e^{i \theta}, \text { with }-\theta_{0}<\theta<\theta_{0}, \quad t \in \mathbf{R}\right\}
$$

is contained in $\Omega$ and the curve $\gamma(t)=\sigma^{-1}\left(e^{c t} w\right)$ converges to a nontangentially as $t$ goes to $+\infty$ for all $w \in V$. Moreover, given a curve $\gamma$ : 
$[0,1) \rightarrow D$ such that $\gamma(r)$ converges to a nontangentially, then there exist $r_{0} \in(0,1)$ and $\varepsilon>0$ such that for all $r \geq r_{0}$, we have that $\sigma(\gamma(r)) \in V$ and $\operatorname{dist}(\sigma(\gamma(r)), \partial V)>\varepsilon|\sigma(\gamma(r))|$.

Proof. Let us denote $\alpha(t)=\varphi_{t}^{\prime}(a)$ for all $t \geq 0$. On the one hand, by the chain rule, we have that $\alpha(t+s)=\alpha(t) \alpha(s)$. On the other hand, $\alpha(t)=$ $\varphi_{t}^{\prime}(a)=\lim _{n \rightarrow \infty} \varphi_{t}^{\prime}\left(\left(1-\frac{1}{n}\right) a\right)$. For each $n$, the function $t \mapsto \varphi_{t}^{\prime}\left(\left(1-\frac{1}{n}\right) a\right)$ is continuous, so $\alpha$ is measurable. Since the only measurables solutions of the functional equation $\alpha(t+s)=\alpha(t) \alpha(s)$ are exponentials, there is $\beta \in$ $(0,+\infty) \cup\{\infty\}$ such that $\varphi_{t}^{\prime}(a)=e^{\beta t}$ for all $t \geq 0$.

Let us consider the invariant subset of $\Omega$ given by

$$
V(\Omega)=\cap_{n \geq 0} e^{-c t} \Omega .
$$

If $V(\Omega)$ is non-void and since it is open, we can write $V(\Omega)$ as the union of the countable family of its different connected components. These components will be denoted by $V_{j}(\Omega)(j \in J)$. It can be proved that given $t>0$, we have that $e^{-c t} V_{j}(\Omega)=V_{j}(\Omega)$ for all $j$. Thus, each $V_{j}(\Omega)$ is a open spiral sector. Now, by [6, Theorem 3.1], given a component $V_{j}(\Omega)$, there is a unique repulsive boundary fixed point $\xi\left(V_{j}(\Omega)\right)$ of $\left(\varphi_{t}\right)$. Moreover the map $\xi$, between the connected components and the repulsive boundary fixed points, is bijective. Moreover, following the proof of [6, Theorem 3.1], it can be checked that the curve $\gamma(t)=\sigma^{-1}\left(e^{c t} w\right)$ converges to $\xi\left(V_{j}(\Omega)\right)$ nontangentially as $t$ goes to $+\infty$ for all $w \in V_{j}(\Omega)$.

Now, take a curve $\gamma:[0,1) \rightarrow \mathrm{D}$ such that $\gamma(r)$ converges to a repulsive boundary fixed point $\xi=\xi\left(V_{j}(\Omega)\right)$ nontangentially. Arguing as in the proof of [6, Claim 4.3], there is a connected component $V_{l}(\Omega)$ such that $\gamma(r) \in V_{l}(\Omega)$ for $r$ large enough. There is $r_{0}$ such that for $r \geq r_{0}$, we have that

$$
\left|\xi-\varphi_{t}(\gamma(r))\right| \leq\left(\varphi_{t}^{\prime}(\xi)+1\right)|\xi-\gamma(r)| .
$$

Therefore,

$$
\left|\gamma(r)-\varphi_{t}(\gamma(r))\right| \leq\left(\varphi_{t}^{\prime}(\xi)+2\right)|\xi-\gamma(r)| .
$$

Hence, estimating the hyperbolic distance along the segment $\left[\gamma(r), \varphi_{t}(\gamma(r))\right]$, we get

$$
\begin{aligned}
\rho_{\mathrm{D}}\left(\gamma(r), \varphi_{t}(\gamma(r))\right) & \leq 2 \int_{\left[\gamma(r), \varphi_{t}(\gamma(r))\right]} \frac{d s(x)}{\operatorname{dist}(x, \partial \mathrm{D})} \\
& \leq 2 \frac{\left(\varphi_{t}^{\prime}(\xi)+2\right)|\xi-\gamma(r)|}{1-|\gamma(r)|} .
\end{aligned}
$$


Since $\gamma(r)$ converges to $\xi=\xi\left(V_{j}(\Omega)\right)$ nontangentially, we have that there is a constant $c$ such that $\frac{|\xi-\gamma(r)|}{1-|\gamma(r)|} \leq c$ for $r$ large enough. So

$$
\rho_{\Omega}\left(\sigma(\gamma(r)), e^{-c t} \sigma(\gamma(r))\right)=\rho_{\mathrm{D}}\left(\gamma(r), \varphi_{t}(\gamma(r))\right) \leq 2\left(\varphi_{t}^{\prime}(\xi)+2\right) c .
$$

Finally, by the Distance Lemma [9, Chapter 9], we obtain that

$$
\begin{aligned}
4\left(\varphi_{t}^{\prime}(\xi)+2\right) c & \geq \log \left(1+\frac{\left|\sigma(\gamma(r))-e^{-c t} \sigma(\gamma(r))\right|}{\min \left\{\operatorname{dist}(\sigma(\gamma(r)), \partial \Omega), \operatorname{dist}\left(e^{-c t} \sigma(\gamma(r)), \partial \Omega\right)\right\}}\right) \\
& \geq \log \left(1+\frac{\left|1-e^{-c t}\right||\sigma(\gamma(r))|}{\operatorname{dist}(\sigma(\gamma(r)), \partial \Omega)}\right) .
\end{aligned}
$$

Therefore, there is $\varepsilon>0$ such that dist $(\sigma(\gamma(r)), \partial \Omega)>\varepsilon|\sigma(\gamma(r))|$ for $r$ large enough. Since $\lim _{r \rightarrow 1} \frac{\operatorname{dist}\left(\sigma(\gamma(r)), V_{l}(\Omega)\right)}{\operatorname{dist}(\sigma(\gamma(r)), \partial \Omega)}=1$, we obtain that dist $\left(\sigma(\gamma(r)), V_{l}(\Omega)\right)>$ $\varepsilon|\sigma(\gamma(r))|$ for $r$ large enough. Finally, by [2, Lemma 17],

$$
\xi=\lim _{r \rightarrow 1} \sigma^{-1}(\sigma(\gamma(r)))=\lim _{t \rightarrow+\infty} \sigma^{-1}\left(e^{c t} w\right)=\xi\left(V_{l}(\Omega)\right)
$$

for all $w \in V_{l}(\Omega)$. That is $j=l$.

Proof of Theorem 1. (2) implies (3). This is general theory (see, for instance, [7, Theorem 10.5]).

(3) implies (1). Denote $\beta=\angle \lim _{z \rightarrow a} G^{\prime}(z) \in \mathrm{C}$. Then $G^{\prime}$ is bounded on the radial segment $[0,1] a$, that is, there is $c$ such that $\left|G^{\prime}(x a)\right| \leq c$ for all $0 \leq x \leq 1$. Since $G(a):=\angle \lim _{z \rightarrow a} G(z)=0$, we have that

$$
\begin{aligned}
|G(x a)|=|G(x a)-G(a)|=\mid & \int_{a}^{x a} G^{\prime}(s) d s \mid \\
& \leq \int_{a}^{x a}\left|G^{\prime}(s)\right| d s \leq c|x a-a|=c(1-x)
\end{aligned}
$$

for all $0 \leq x<1$. Since the infinitesimal generator satisfies that $\frac{\partial \varphi_{t}(z)}{\partial t}=$ $G(z) \varphi_{t}^{\prime}(z)$ and $\varphi_{t}(\mathrm{D}) \subset \mathrm{D}$, we see that

$$
\begin{aligned}
\left|\frac{\partial \varphi_{t}}{\partial t}(x a)\right| & =|G(x a)|\left|\varphi_{t}^{\prime}(x a)\right| \leq c(1-x)\left|\varphi_{t}^{\prime}(x a)\right| \\
& \leq c(1-x) \frac{1-\left|\varphi_{t}(x a)\right|^{2}}{1-x^{2}} \leq 2 c\left(1-\left|\varphi_{t}(x a)\right|\right) .
\end{aligned}
$$

Therefore,

$$
\left|\frac{\partial}{\partial t} \log \left(1-\bar{a} \varphi_{t}(x a)\right)\right|=\frac{\left|\frac{\partial \varphi_{t}}{\partial t}(x a)\right|}{\left|1-\bar{a} \varphi_{t}(x a)\right|} \leq 2 c .
$$


Moreover, since $\varphi_{0}(x a)=x a$, we obtain that

$$
\left|\log \left(1-\bar{a} \varphi_{t}(x a)\right)-\log (1-x)\right| \leq 2 c t .
$$

This implies that $\varphi_{t}(a)=a$ for all $t$ and

$$
\left|\arg \left(1-\bar{a} \varphi_{t}(x a)\right)\right| \leq \frac{\pi}{4} \quad \text { for all } \quad 0<x<1, \quad 0 \leq t \leq \frac{\pi}{8 c} .
$$

Since the infinitesimal generator satisfies that $\frac{\partial \varphi_{t}(z)}{\partial t}=G\left(\varphi_{t}(z)\right)$, we have that $\frac{\partial}{\partial z}\left(\frac{\partial \varphi_{t}(z)}{\partial t}\right)=G^{\prime}\left(\varphi_{t}(z)\right) \varphi_{t}^{\prime}(z)$ and, therefore,

$$
\frac{\partial}{\partial t} \log \left(\varphi_{t}^{\prime}(z)\right)=\frac{\frac{\partial}{\partial z}\left(\frac{\partial \varphi_{t}(z)}{\partial t}\right)}{\varphi_{t}^{\prime}(z)}=G^{\prime}\left(\varphi_{t}(z)\right) .
$$

Now, take $0 \leq t \leq \frac{\pi}{8 c}$ and $\Delta$ a Stolz sector with vertex $a$ and angle $\frac{\pi}{4}$. We have that $\varphi_{t}(x a) \in \Delta$ for all $0<x<1$ and for all $0 \leq t \leq \frac{\pi}{8 c}$. So $\varphi_{t}(x a)$ converges nontangentially to $a$ as $x$ goes to 1 and, therefore, $G^{\prime}\left(\varphi_{t}(x a)\right)$ converges to $\beta$ as $x$ goes to 1 . Finally, bearing in mind that $G^{\prime}$ is bounded on $\Delta \cup\{a\}$ and the Dominated Convergence Theorem, we have

$$
\log \left(\varphi_{t}^{\prime}(x a)\right)-\beta t=\int_{0}^{t}\left(G^{\prime}\left(\varphi_{s}(x a)\right)-\beta\right) d s \rightarrow 0
$$

as $x \rightarrow 1$. That is, $\varphi_{t}^{\prime}(a)=e^{\beta t}$ for all $0 \leq t \leq \frac{\pi}{8 c}$. But this implies that $\varphi_{t}^{\prime}(a)=e^{\beta t}$ for all $t \geq 0$ and, in particular, $\beta \in \mathrm{R}$.

(1) implies (2). When $a$ is the Denjoy-Wolff point, the result was proved by Shoikhet [10, Proposition 4.6.2].

Now, suppose that $a$ is a repulsive fixed point. Firstly, we assume that the Denjoy-Wolff point is equal to 1 . By Lemma 1 , we know that $\varphi_{t}^{\prime}(a)=e^{\beta t}$ for all $t \geq 0$. Take $V$ the open strip given by that lemma and consider the Riemann map from $D$ onto the strip $V$ given by

$$
k(z)=\frac{\beta}{\pi} \log \left(\frac{1-\bar{a} z}{1+\bar{a} z}\right)+c i
$$

for all $z \in \mathrm{D}$. Notice that $k(r a)=\frac{\beta}{\pi} \log \left(\frac{1-r}{1+r}\right)+c i$ and so the curve $r \mapsto$ $\sigma^{-1}(k \underset{\sim}{(r a))}$ converges to $a$ nontangentially as $r$ goes to 1 .

Set $\widetilde{\Omega}=\sigma^{-1}(V) \subset \mathrm{D}$. Then the map $g=\sigma^{-1} \circ k$ is a Riemann map from D onto $\widetilde{\Omega}$ and $a$ is a fixed point of $g$. In particular, there exists the angular derivative of $g$ at $a$ and $g^{\prime}(a) \in(0,+\infty]$. Moreover, using Lemma 1, we have

Claim. Given a curve $\gamma:[0,1) \rightarrow \mathrm{D}$ such that $\gamma(r)$ converges to a nontangentially, then there are a number $r_{0} \in(0,1)$ and $\varepsilon>0$ such that for 
all $r \geq r_{0}$ we have that $\gamma(r) \in \widetilde{\Omega}$ and $g^{-1}(\gamma(r))=k^{-1} \circ \sigma(\gamma(r))$ converge to a nontangentially.

Since $V+t=V$ for all $t$, we get that $\varphi_{t}(\widetilde{\Omega})=\widetilde{\Omega}$, so the semigroup of analytic functions in the unit disc given by $\psi_{t}(z)=g^{-1} \circ \varphi_{t} \circ g(z)$ is welldefined. Let us denote by $F$ the infinitesimal generator of the semigroup $\left(\psi_{t}\right)$. Hence,

$$
\frac{\partial \psi_{t}(z)}{\partial t}=\frac{1}{g^{\prime}\left(g^{-1} \circ \varphi_{t} \circ g(z)\right)} \frac{\partial \varphi_{t}(g(z))}{\partial t} g^{\prime}(z) .
$$

In particular, for $t=0$,

$$
F(z)=G(g(z)) \quad \text { for all } \quad z \in \mathrm{D} .
$$

Now, notice that

$$
\begin{aligned}
\psi_{t}(z) & =k^{-1} \circ \sigma \circ \varphi_{t} \circ \sigma^{-1} \circ k(z) \\
& =k^{-1} \circ \sigma \circ \sigma^{-1}\left(\sigma\left(\sigma^{-1} \circ k(z)\right)+t\right)=k^{-1}(k(z)+t)
\end{aligned}
$$

for all $t \geq 0$ and $z \in \mathrm{D}$. Therefore,

$$
F(z)=\frac{1}{k^{\prime}(z)}=\frac{\pi}{2 \beta a}\left(z^{2}-a^{2}\right)
$$

and

$$
G(g(z))=\frac{\pi}{2 \beta a}\left(z^{2}-a^{2}\right) \quad \text { for all } \quad z \in \mathrm{D} .
$$

Take a curve $\gamma:[0,1) \rightarrow \mathrm{D}$ such that $\gamma(r)$ converges to $a$ nontangentially. By the claim, there are a number $r_{0} \in(0,1)$ and $\varepsilon>0$ such that for all $r \geq r_{0}$, we have that $\gamma(r) \in \widetilde{\Omega}$ and $g^{-1}(\gamma(r))=k^{-1} \circ \sigma(\gamma(r))$ converge to $a$ nontangentially. On the one hand, we have

$$
G(\gamma(r))=F\left(g^{-1}(\gamma(r))\right)=-\frac{\pi}{2 \beta a}\left(a^{2}-g^{-1}(\gamma(r))^{2}\right) .
$$

So, $\angle \lim _{z \rightarrow a} G(z)=0$. On the other hand, we have

$$
\begin{aligned}
\frac{G(\gamma(r))-G(a)}{\gamma(r)-a} & =\frac{\pi}{2 \beta a} \frac{\left(g^{-1}(\gamma(r))^{2}-a^{2}\right)}{\gamma(r)-a} \\
& =\frac{\pi}{2 \beta a} \frac{\left(g^{-1}(\gamma(r))-a\right)}{\gamma(r)-a}\left(g^{-1}(\gamma(r))+a\right) \\
& =\frac{\pi}{2 \beta a} \frac{1}{\frac{g\left(g^{-1}(\gamma(r))\right)-a}{g^{-1}(\gamma(r))-a}}\left(g^{-1}(\gamma(r))+a\right) .
\end{aligned}
$$


Since $g^{-1}(\gamma(r))$ converge to $a$ nontangentially, we deduce

$$
\lim _{r \rightarrow 1} \frac{g\left(g^{-1}(\gamma(r))\right)-a}{g^{-1}(\gamma(r))-a}=g^{\prime}(a) \in(0,+\infty] .
$$

Therefore, $\lim _{r \rightarrow 1} \frac{G(\gamma(r))-G(a)}{\gamma(r)-a}=\frac{\pi}{\beta} \frac{1}{g^{\prime}(a)} \in[0,+\infty)$. Hence

$$
\angle \lim _{z \rightarrow a} \frac{G(z)}{z-a}=\frac{\pi}{\beta} \frac{1}{g^{\prime}(a)} \in[0,+\infty) .
$$

Now, suppose that the Denjoy-Wolff point is zero. Then, by Lemma 3, there is $\beta \in(0,+\infty)$ such that $\varphi_{t}^{\prime}(a)=e^{\beta t}$ for all $t \geq 0$ and take

$$
V=\left\{w \in \mathrm{C}: w=d e^{-c t} e^{i \theta}, \text { with }-\theta_{0}<\theta<\theta_{0}, t \in \mathbf{R}\right\}
$$

the open spiral sector given in the lemma. Consider the Riemann map from D onto the $V$ given by

$$
k(z)=d \exp \left[\frac{2}{\pi} \theta_{0} \frac{\operatorname{Re}(c)}{|c|^{2}} c \log \left(\frac{1+\bar{a} z}{1-\bar{a} z}\right)\right]
$$

for all $z \in \mathrm{D}$. Notice that $k(r a)=d \exp \left[\frac{2}{\pi} \theta_{0} \frac{\operatorname{Re}(c)}{|c|^{2}} c \log \left(\frac{1+r}{1-r}\right)\right]$, so the curve $r \mapsto \sigma^{-1}(k(r a))$ converges to $a$ nontangentially as $r$ goes to 1 .

Set $\widetilde{\Omega}=\sigma^{-1}(V) \subset \mathrm{D}$. Then the map $g=\sigma^{-1} \circ k$ is a Riemann map from D onto $\widetilde{\Omega}$ and $a$ is a fixed point of $g$. In particular, there exists the angular derivative of $g$ at $a$ and $g^{\prime}(a) \in(0,+\infty)$. Moreover, by Lemma 3, we have

Claim. Given a curve $\gamma:[0,1) \rightarrow \mathrm{D}$ such that $\gamma(r)$ converges to a nontangentially, then there are a number $r_{0} \in(0,1)$ and $\varepsilon>0$ such that for all $r \geq r_{0}$, we have that $\gamma(r) \in \widetilde{\Omega}$ and $g^{-1}(\gamma(r))=k^{-1} \circ \sigma(\gamma(r))$ converge to a nontangentially.

The rest of the computations is similar to the case when the Denjoy-Wolff point is in the boundary, but for the sake of completeness we detail them.

Since $e^{-c t} V=V$ for all $t$, we see that $\varphi_{t}(\widetilde{\Omega})=\widetilde{\Omega}$, so the semigroup of analytic functions in the unit disc given by $\psi_{t}(z)=g^{-1} \circ \varphi_{t} \circ g(z)$ is welldefined. Let us denote by $F$ the infinitesimal generator of the semigroup $\left(\psi_{t}\right)$. Hence

$$
\frac{\partial \psi_{t}(z)}{\partial t}=\frac{1}{g^{\prime}\left(g^{-1} \circ \varphi_{t} \circ g(z)\right)} \frac{\partial \varphi_{t}(g(z))}{\partial t} g^{\prime}(z) \text {. }
$$

In particular, for $t=0$,

$$
F(z)=G(g(z)) \quad \text { for all } \quad z \in \mathrm{D} .
$$


Notice that

$$
\psi_{t}(z)=k^{-1} \circ \sigma \circ \varphi_{t} \circ \sigma^{-1} \circ k(z)=k^{-1}\left(e^{-c t} k(z)\right)
$$

for all $t \geq 0$ and $z \in \mathrm{D}$. Therefore,

$$
F(z)=-c \frac{k(z)}{k^{\prime}(z)}=\frac{\pi|c|^{2}}{4 \theta_{0} \operatorname{Re}(c) a}\left(z^{2}-a^{2}\right)
$$

and

$$
G(g(z))=\frac{\pi|c|^{2}}{4 \theta_{0} \operatorname{Re}(c) a}\left(z^{2}-a^{2}\right) \quad \text { for all } \quad z \in \mathrm{D} .
$$

Now, take a curve $\gamma:[0,1) \rightarrow \mathrm{D}$ such that $\gamma(r)$ converges to $a$ nontangentially. By the claim, there are a number $r_{0} \in(0,1)$ and $\varepsilon>0$ such that for all $r \geq r_{0}$, we have that $\gamma(r) \in \widetilde{\Omega}$ and $g^{-1}(\gamma(r))=k^{-1} \circ \sigma(\gamma(r))$ converge to $a$ nontangentially. On the one hand, we have

$$
G(\gamma(r))=F\left(g^{-1}(\gamma(r))\right)=\frac{\pi|c|^{2}}{4 \theta_{0} \operatorname{Re}(c) a}\left(g^{-1}(\gamma(r))^{2}-a^{2}\right) .
$$

Thus, $\angle \lim _{z \rightarrow a} G(z)=0$. On the other hand, we have

$$
\begin{aligned}
\frac{G(\gamma(r))-G(a)}{\gamma(r)-a} & =\frac{\pi|c|^{2}}{4 \theta_{0} \operatorname{Re}(c) a} \frac{\left(g^{-1}(\gamma(r))^{2}-a^{2}\right)}{\gamma(r)-a} \\
& =\frac{\pi|c|^{2}}{4 \theta_{0} \operatorname{Re}(c) a} \frac{\left(g^{-1}(\gamma(r))-a\right)}{\gamma(r)-a}\left(g^{-1}(\gamma(r))+a\right) \\
& =\frac{\pi|c|^{2}}{4 \theta_{0} \operatorname{Re}(c) a} \frac{1}{\frac{g\left(g^{-1}(\gamma(r))\right)-a}{g^{-1}(\gamma(r))-a}}\left(g^{-1}(\gamma(r))+a\right) .
\end{aligned}
$$

Since $g^{-1}(\gamma(r))$ converge to $a$ nontangentially, we deduce

$$
\lim _{r \rightarrow 1} \frac{g\left(g^{-1}(\gamma(r))\right)-a}{g^{-1}(\gamma(r))-a}=g^{\prime}(a) \in(0,+\infty] .
$$

Therefore, $\lim _{r \rightarrow 1} \frac{G(\gamma(r))-G(a)}{\gamma(r)-a}=\frac{\pi|c|^{2}}{2 \theta_{0} \operatorname{Re}(c)} \frac{1}{g^{\prime}(a)} \in[0,+\infty)$. Hence

$$
\angle \lim _{z \rightarrow a} \frac{G(z)}{z-a}=\frac{\pi|c|^{2}}{2 \theta_{0} \operatorname{Re}(c)} \frac{1}{g^{\prime}(a)} \in[0,+\infty) .
$$

Proof of Theorem 2. We know that

$$
G(z)=(b-z)(1-\bar{b} z) p(z),
$$


for all $z \in \mathrm{D}$, where $p: \mathrm{D} \rightarrow \mathrm{C}$ is an analytic function with $\operatorname{Re} p \geq 0$. By Theorem 1, it follows

$$
\angle \lim _{z \rightarrow \xi_{j}} G(z)=0 \quad \text { and } \quad \angle \lim _{z \rightarrow \xi_{j}} G^{\prime}(z)=\beta_{j} \in(0,+\infty)
$$

for all $j=1,2, \ldots, n$. Therefore,

$$
\angle \lim _{z \rightarrow \xi_{j}} p(z)=0 \quad \text { and } \quad \angle \lim _{z \rightarrow \xi_{j}} p^{\prime}(z)=\frac{\beta_{j}}{\left(b-\xi_{j}\right)\left(1-\bar{b} \xi_{j}\right)},
$$

for all $j=1,2, \ldots, n$. Now, consider the semigroup of analytic functions $\left(\psi_{t}\right)$ whose infinitesimal generator is $F(z)=-z p(z)$. Then the Denjoy-Wolff point of the function $\psi_{t}$ is zero for all $t$,

$$
\angle \lim _{z \rightarrow \xi_{j}} F(z)=\angle \lim _{z \rightarrow \xi_{j}}(-z p(z))=0
$$

and

$$
\angle \lim _{z \rightarrow \xi_{j}} F^{\prime}(z)=-\angle \lim _{z \rightarrow \xi_{j}} z p^{\prime}(z)=-\frac{\xi_{j} \beta_{j}}{\left(b-\xi_{j}\right)\left(1-\bar{b} \xi_{j}\right)}=\frac{\beta_{j}}{2-2 \operatorname{Re}\left(\bar{b} \xi_{j}\right)}
$$

for all $j=1,2, \ldots, n$. Again, we apply Theorem 1 , but this time to the semigroup $\left(\psi_{t}\right)$, and we obtain that $\xi_{j}$ is a repulsive fixed point of $\psi_{t}$ and $\psi_{t}^{\prime}\left(\xi_{j}\right)=e^{\alpha_{j} t}$, where $\alpha_{j}:=\angle \lim _{z \rightarrow \xi_{j}} F^{\prime}(z)=\frac{\beta_{j}}{2-2 \operatorname{Re}\left(\bar{b} \xi_{j}\right)}$. Now, by [4, Theorem 7.1], we have that

$$
\sum_{j=1}^{n} \frac{1}{\log \psi_{t}^{\prime}\left(\xi_{j}\right)} \leq 2 \operatorname{Re} \frac{1}{B}
$$

where $B=\lim _{r \rightarrow 1^{-}} \log \frac{\psi_{t}\left(r \xi_{j}\right)}{\psi_{t}^{\prime}(0) r \xi_{j}}$ (this limit is independent of $j$, we choose the branch of $\log \frac{\psi_{t}(z)}{\psi_{t}^{\prime}(0) z}$ that is zero at $\left.z=0\right)$. The proof of [4, Theorem 7.1] shows that $B=p(0) t$. Then

$$
\sum_{j=1}^{n} \frac{2-2 \operatorname{Re}\left(\bar{b} \xi_{j}\right)}{\beta_{j} t}=\sum_{j=1}^{n} \frac{1}{\alpha_{j} t}=\sum_{j=1}^{n} \frac{1}{\log \psi_{t}^{\prime}\left(\xi_{j}\right)} \leq 2 \operatorname{Re} \frac{1}{B}=2 \operatorname{Re} \frac{1}{p(0) t}
$$

That is,

$$
\sum_{j=1}^{n} \frac{1-\operatorname{Re}\left(\bar{b} \xi_{j}\right)}{\beta_{j}} \leq \operatorname{Re} \frac{1}{p(0)}=\operatorname{Re} \frac{1}{\bar{b} G(0)}
$$
if

Again by [4, Theorem 7.1], equality holds in this last inequality if and only

$$
\psi_{t}(z)=\widetilde{\sigma}^{-1}\left(e^{-p(0) t} \widetilde{\sigma}(z)\right)
$$


where

$$
\tilde{\sigma}(z)=z \prod_{j=1}^{n}\left(1-\overline{\xi_{j}} z\right)^{-\frac{B}{\log \psi_{t}^{\prime}\left(\xi_{j}\right)}}=z \prod_{j=1}^{n}\left(1-\overline{\xi_{j}} z\right)^{-\frac{2 p(0)\left(1-\operatorname{Re}\left(\overline{b_{j}}\right)\right)}{\beta_{j}}} .
$$

Therefore,

$$
\begin{aligned}
-z p(z)=F(z)=\left.\frac{\partial \psi_{t}(z)}{\partial t}\right|_{t=0} & =-p(0) \frac{\tilde{\sigma}(z)}{\widetilde{\sigma}^{\prime}(z)} \\
& =\frac{-p(0) z}{1+2 p(0) z \sum_{j=1}^{n} \frac{\left(1-\operatorname{Re}\left(\bar{b} \xi_{j}\right)\right)}{\beta_{j}} \frac{\overline{\xi_{j}}}{1-\overline{\xi_{j} z}}} .
\end{aligned}
$$

That is, if the equality holds, then the infinitesimal generator of the semigroup $\left(\varphi_{t}\right)$ is

$$
G(z)=\bar{b}(b-z)^{2} p(z)=\frac{\bar{b}(b-z)^{2} p(0)}{1+2 p(0) z \sum_{j=1}^{n} \frac{\left(1-\operatorname{Re}\left(\bar{b} \xi_{j}\right)\right)}{\beta_{j}} \frac{\overline{\xi_{j}}}{1-\overline{\xi_{j}} z} .} .
$$

Bearing in mind that $\varphi_{t}(z)=\sigma^{-1}(\sigma(z)+t)$, we have $G(z)=\left.\frac{\partial \varphi_{t}(z)}{\partial t}\right|_{t=0}=$ $\frac{1}{\sigma^{\prime}(z)}$. That is,

$$
\sigma^{\prime}(z)=\frac{b}{(b-z)^{2} p(0)}+2 z \sum_{j=1}^{n} \frac{\left(1-\operatorname{Re}\left(\bar{b} \xi_{j}\right)\right)}{\beta_{j}} \frac{\overline{\xi_{j}}}{1-\overline{\xi_{j}} z} \frac{\bar{b}}{(1-\bar{b} z)^{2}} .
$$

Finally, taking in account that $\sigma(0)=0$, we get

$$
\begin{aligned}
\sigma(z)= & \frac{z}{(b-z) p(0)} \\
& +2 \sum_{j=1}^{n} \frac{\left(1-\operatorname{Re}\left(\bar{b} \xi_{j}\right)\right.}{\beta_{j}}\left[\frac{\xi_{j} \bar{b}}{\left(1-\bar{b} \xi_{j}\right)^{2}} \log \left[\frac{1-\bar{b} z}{1-\overline{\xi_{j}} z}\right]+\frac{\overline{\xi_{j}}}{1-\overline{\xi_{j}} b} \frac{z}{1-\bar{b} z}\right] .
\end{aligned}
$$

Proof of Theorem 3. Consider the function

$$
p(z)=\frac{2 a}{a-\lambda} \frac{\varphi(z)-\lambda}{a-\varphi(z)} \quad \text { for all } z \in \mathrm{D} .
$$

It is easy to check that $p$ satisfies the following five properties

a: $\operatorname{Re} p(z)>0$ for all $z \in \mathrm{D}$,

b: $\angle \lim _{z \rightarrow b} p(z)=\infty$, 


$$
\begin{aligned}
& \mathrm{c}: \angle \lim _{z \rightarrow b}(\bar{b} z-1) p(z)=\frac{-2}{b \bar{a} \varphi^{\prime}(b)} \in(-\infty, 0), \\
& \mathrm{d}: \angle \lim _{z \rightarrow z_{j}} p(z)=0 \text { for } j=1, \ldots, n, \\
& \mathrm{e}: \angle \lim _{z \rightarrow z_{j}} \frac{p(z)}{z-z_{j}}=\frac{2 a}{(a-\lambda)^{2}} \varphi^{\prime}\left(z_{j}\right) .
\end{aligned}
$$

Now, we introduce the function $G(z)=b(\bar{b} z-1)^{2} p(z)$ for all $z \in \mathrm{D}$. Then $G$ is the infinitesimal generator of a semigroup of analytic functions $\left(\varphi_{t}\right)$ with Denjoy-Wolff point $b \in \partial \mathrm{D}$. Moreover, by (c),

$$
\angle \lim _{z \rightarrow b} \frac{G(z)}{z-b}=\angle \lim _{z \rightarrow b}(\bar{b} z-1) p(z)=\frac{-2}{b \bar{a} \varphi^{\prime}(b)}=\frac{-2}{\left|\varphi^{\prime}(b)\right|} \in(-\infty, 0) .
$$

So, by Theorem 1, $\varphi_{t}^{\prime}(b)=\exp \left(\frac{-2}{\left|\varphi^{\prime}(b)\right|} t\right)$ for all $t$. What is more, by (d) and (e), we have

$$
\angle \lim _{z \rightarrow z_{j}} G(z)=b\left(\bar{b} z_{j}-1\right)^{2}\left(\angle \lim _{z \rightarrow z_{j}} p(z)\right)=0
$$

and

$$
\begin{aligned}
\angle \lim _{z \rightarrow z_{j}} \frac{G(z)}{z-z_{j}} & =b\left(\bar{b} z_{j}-1\right)^{2}\left(\angle \lim _{z \rightarrow z_{j}} \frac{p(z)}{z-z_{j}}\right)=b\left(\bar{b} z_{j}-1\right)^{2} \frac{2 a}{(a-\lambda)^{2}} \varphi^{\prime}\left(z_{j}\right) \\
& =2 \frac{\left(z_{j}-b\right)^{2} \bar{b} \overline{z_{j}}}{(a-\lambda)^{2} \bar{a} \bar{\lambda}} z_{j} \varphi^{\prime}\left(z_{j}\right)=2 \frac{1-\operatorname{Re}\left(\bar{b} z_{j}\right)}{1-\operatorname{Re}(\bar{\lambda} a)} \bar{\lambda} z_{j} \varphi^{\prime}\left(z_{j}\right) \\
& =2 \frac{1-\operatorname{Re}\left(\bar{b} z_{j}\right)}{1-\operatorname{Re}(\bar{\lambda} a)}\left|\varphi^{\prime}\left(z_{j}\right)\right|
\end{aligned}
$$

for all $j=1,2, \ldots, n$. Therefore, again by Theorem 1 , we have that $z_{j}$ is a fixed point of $\left(\varphi_{t}\right)$ and $\varphi_{t}^{\prime}\left(z_{j}\right)=\exp \left(2 \frac{1-\operatorname{Re}\left(\bar{b} z_{j}\right)}{1-\operatorname{Re}(\bar{\lambda} a)}\left|\varphi^{\prime}\left(z_{j}\right)\right| t\right)$ for all $t$. Now, by $[4$, Theorem 6.1], we obtain

$$
\sum_{j=1}^{n} \frac{1}{2 \frac{1-\operatorname{Re}\left(\bar{b} z_{j}\right)}{1-\operatorname{Re}(\bar{\lambda} a)}\left|\varphi^{\prime}\left(z_{j}\right)\right|} \leq \frac{\left|\varphi^{\prime}(b)\right|}{2}
$$

That is

$$
\sum_{j=1}^{n} \frac{1-\operatorname{Re}(\bar{\lambda} a)}{1-\operatorname{Re}\left(\bar{b} z_{j}\right)} \frac{1}{\left|\varphi^{\prime}\left(z_{j}\right)\right|} \leq\left|\varphi^{\prime}(b)\right|
$$

Again by [4, Theorem 6.1], the equality holds in this last inequality if and only if

$$
\varphi_{t}(z)=\sigma^{-1}(\sigma(z)+t)
$$


where

$$
\sigma(z)=\frac{1}{2} \sum_{j=1}^{n} \frac{1-\operatorname{Re}(\bar{\lambda} a)}{\left(1-\operatorname{Re}\left(\bar{b} z_{j}\right)\right)\left|\varphi^{\prime}\left(z_{j}\right)\right|} \log \left[\frac{b+z}{b-z}-\frac{b+z_{j}}{b-z_{j}}\right]
$$

In this case, $G(z)=\frac{1}{\sigma^{\prime}(z)}$. Since

$$
\sigma^{\prime}(z)=(a-\lambda)^{2} \bar{\lambda} \bar{a} b^{2} \sum_{j=1}^{n} \frac{z_{j}}{\left|\varphi^{\prime}\left(z_{j}\right)\right|} \frac{1}{\left(z^{2}-b^{2}\right)\left(b-z_{j}\right)^{2}-\left(z_{j}^{2}-b^{2}\right)(b-z)^{2}}
$$

we compute

$$
\begin{aligned}
p(z) & =\frac{G(z)}{b(\bar{b} z-1)^{2}}=\frac{1}{b(\bar{b} z-1)^{2} \sigma^{\prime}(z)} \\
& =\frac{1}{b(z-b)^{2}(a-\lambda)^{2} \bar{\lambda} \bar{a} \sum_{j=1}^{n} \frac{z_{j}}{\left|\varphi^{\prime}\left(z_{j}\right)\right|} \frac{1}{\left(z^{2}-b^{2}\right)\left(b-z_{j}\right)^{2}-\left(z_{j}^{2}-b^{2}\right)(b-z)^{2}}} \\
& =\frac{1}{b(z-b)(a-\lambda)^{2} \bar{\lambda} \bar{a} \sum_{j=1}^{n} \frac{z_{j}}{\left|\varphi^{\prime}\left(z_{j}\right)\right|} \frac{1}{\left(b-z_{j}\right)} \frac{1}{(z+b)\left(b-z_{j}\right)-\left(z_{j}+b\right)(b-z)}} \\
& =\frac{2}{(z-b)(a-\lambda)^{2} \bar{\lambda} \bar{a} \sum_{j=1}^{n} \frac{z_{j}}{\left|\varphi^{\prime}\left(z_{j}\right)\right|} \frac{1}{b-z_{j}} \frac{1}{z-z_{j}}} .
\end{aligned}
$$

But $p(z)=\frac{2 a}{a-\lambda} \frac{\varphi(z)-\lambda}{a-\varphi(z)}$, so

$$
\begin{aligned}
\varphi(z) & =\frac{2 \lambda+(a-\lambda) p(z)}{2 a+(a-\lambda) p(z)} \\
& =\frac{\lambda(z-b)(a-\lambda)^{2} \bar{\lambda} \bar{a} \sum_{j=1}^{n} \frac{z_{j}}{\left|\varphi^{\prime}\left(z_{j}\right)\right|} \frac{1}{b-z_{j}} \frac{1}{z-z_{j}}+(a-\lambda)}{a(z-b)(a-\lambda)^{2} \bar{\lambda} \bar{a} \sum_{j=1}^{n} \frac{z_{j}}{\left|\varphi^{\prime}\left(z_{j}\right)\right|} \frac{1}{b-z_{j}} \frac{1}{z-z_{j}}+(a-\lambda)} \\
& =\frac{(a-\lambda) \lambda \sum_{j=1}^{n} \frac{z_{j}}{\left|\varphi^{\prime}\left(z_{j}\right)\right|} \frac{1}{b-z_{j}} \frac{z-b}{z-z_{j}}+a \lambda}{(a-\lambda) a \sum_{j=1}^{n} \frac{z_{j}}{\left|\varphi^{\prime}\left(z_{j}\right)\right|} \frac{1}{b-z_{j}} \frac{z-b}{z-z_{j}}+a \lambda} \\
& =\frac{1+(1-\lambda \bar{a}) \sum_{j=1}^{n} \frac{z_{j}}{\left|\varphi^{\prime}\left(z_{j}\right)\right|} \frac{1}{b-z_{j}} \frac{z-b}{z-z_{j}}}{1-(1-\lambda \bar{a}) \sum_{j=1}^{n} \frac{z_{j}}{\left|\varphi^{\prime}\left(z_{j}\right)\right|} \frac{1}{b-z_{j}} \frac{z-b}{z-z_{j}}},
\end{aligned}
$$

for all $z \in \mathrm{D}$. 


\section{REFERENCES}

1. Berkson, E., and Porta, H., Semigroups of analytic functions and composition operators, Michigan Math. J. 25 (1978), 101-115.

2. Contreras, M. D., and Díaz-Madrigal, S., Analytic flows in the unit disk: angular derivatives and boundary fixed points, Pacific J. Math. 222 (2005), 253-286.

3. Contreras, M. D., Díaz-Madrigal, S., and Pommerenke, Ch., Fixed points and boundary behaviour of the Koenigs function, Ann. Acad. Sci. Fenn. Math. 29 (2004), 471-488.

4. Cowen, C. C., and Pommerenke, Ch., Inequalities for the angular derivative of an analytic function in the unit disk, J. London Math. Soc. (2) 26 (1982), 271-289.

5. Li, K. Y., Inequalities for fixed points of holomorphic functions, Bull. London Math. Soc. 22 (1990), 446-452.

6. Poggi-Corradini, P., Angular derivatives at boundary fixed points for self-maps of the disk, Proc. Amer. Math. Soc. 126 (1998), 1697-1708.

7. Pommerenke, Ch., Univalent Functions, Vandenhoeck \& Ruprecht, Göttingen, 1975.

8. Pommerenke, Ch., Boundary Behaviour of Conformal Maps, Springer-Verlag, Berlin, 1992.

9. Shapiro, J. H., Composition Operators and Classical Function Theory, Springer-Verlag, New York, 1993.

10. Shoikhet, D., Semigroups in Geometrical Function Theory, Kluwer Academic Publishers, Dordrecht, 2001.

CAMINO DE LOS DESCUBRIMIENTOS, S/N DEPARTAMENTO DE MATEMÁTICA APLICADA II ESCUELA SUPERIOR DE INGENIEROS

UNIVERSIDAD DE SEVILLA

41092, SEVILLA

SPAIN

E-mail: contreras@esi.us.es

INSTITUT FÜR MATHEMATIK

TECHNISCHE UNIVERSITÄT

D-10623, BERLIN

GERMANY

E-mail: pommeren@math.tu-berlin.de
CAMINO DE LOS DESCUBRIMIENTOS, S/N DEPARTAMENTO DE MATEMÁTICA APLICADA II ESCUELA SUPERIOR DE INGENIEROS UNIVERSIDAD DE SEVILLA 41092, SEVILLA

SPAIN

E-mail: madrigal@us.es 